\title{
From LiDAR data towards HBIM for energy analysis.
}

Lucía Díaz-Vilariño, Pawel Boguslawski, Miguel Azenha, Lamine Mahdjoubi, Paulo B. Lourenço, Pedro Arias

\begin{abstract}
Knowledge about heritage buildings and structures is interesting for a wide variety of stakeholders, ranging from architects to operators or the public administration. Such knowledge includes a large variety of physical and functional characteristics of the building. Laser scanner allows efficient and accurate 3D digitalization of heritage sites and subsequent data processing towards the creation of geometrically and semantically rich models.
\end{abstract}

Parameterization and semantic enrichment of heritage building representations towards the creation of Heritage Building Information Models (HBIM) generally involves the use of point clouds and images as templates for manual mapping procedures in commercial software. Indeed the information to include in BIM models depends on the requirements of the application it is intended to serve. In view of that, the applications presented in this chapter pertain to automated techniques that were implemented to parameterize point clouds towards models suitable for energy analysis purposes.

The challenge in automating the reconstruction of heritage buildings is to deal with their geometrical complexity and irregularity, meaning that the methodologies selected should be robust and efficient under these conditions. The resulting 3D semantically rich model enhances the knowledge of the heritage building, complementing other representations of the facility such as point clouds or hand-made HBIM. The procedure is implemented and validated in a real case study: the Ducal Palace in Guimarães (Portugal).

\section{Introduction}

For a long time, drawings, schedules or reports generated in traditional ways have been the standard communication between building stakeholders, while 3D modelling was primarily used as a rendering tool, not as a project representation. The popularization of Building Information Modelling (BIM) in the early 2000s (Autodesk, 2002) established the beginning of a new way of thinking in the Architecture, Engineering, Construction and Facility Management (AECFM) industry. BIM encloses the digital representation of both physical and functional characteristics of a facility, representing a shared knowledge resource as a tool for decision making throughout the entire building lifecycle (Eastman et al., 2008).

BIM was initially centred in the support to design and construction processes and consequently, its major uses and research were concentrated in preplanning, design, construction and project delivery (Volk, Stengel and Schultmann, 2014). However, the large amount of existing buildings and the need for knowledge about the built environment has motivated the AECFM industry to put efforts in the latest building lifecycle stages. BIM for existing buildings (EBIM) has emerged in the recent years as a new research trend. Quality control and monitoring under renovation processes (Boschè et al., 2015), energy 
analysis (Díaz-Vilariño et al., 2013), indoor planning and navigation for building crisis management and emergency responses (Isikdag Zlatanova and Underwood, 2013) are just some of the applications that make BIM reconstruction for existing buildings necessary.

The preservation of architectural knowledge is another application of BIM modelling. The DURAARK project (Duraark, 2015), specifically deals with the semantical enrichment of 3D architectural models for the digital long-term preservation of built buildings. This purpose is especially relevant in the case of heritage buildings due to their singularity and value. The modelling of Heritage BIM (HBIM) is a specific domain within EBIM, which requires an accurate and complete documentation of the building, comprising both tangible and intangible knowledge (Simeone et al., 2014).

Non-destructive techniques such as laser scanning and photogrammetry have been widely used in the 3D digitalization of heritage because they allow efficient and accurate records of complex buildings. Even though point clouds are rather accurate, they are composed of massive raw information that requires post processing in order to be converted to parametric components. For instance, a point cloud with several thousands of $3 \mathrm{D}$ points describing a façade is suitable for visualization, while it is not often suited for building analysis or planning.

The level of detail (LoD) and/or different levels of development of a BIM representation is still a controversial topic. Volk, Stengel and Schultmann (2014) consider that BIM can be seen from a narrow and a broader perspective. From a narrow perspective, BIM can be represented attending to the LoD required for the applications it is intended to serve. From a broader perspective, functional, organizational and legal issues should be included in a BIM representation. The same controversy applies for HBIM. Independently of this controversy, building information, both geometry and semantics, should be ultimately processed to ensure its usability for an application. For instance, a curved wall can be accurately surveyed and parameterized but it should be modelled as set of continuous planar polygons for energy analysis purposes.

This chapter specifically deals with a technique for highly automated reconstruction of façade components of heritage buildings from point clouds, with the final goal for application in energy analysis software. The result is a semantically rich $3 \mathrm{D}$ model represented under the paradigm of BIM, including both geometric and semantic information of the building as-is. Point cloud scans are processed by applying some rules in order to obtain parameterized models according to the Green Building XML (gbXML) schema (gbXML, 2015). The development of streamlined processes for obtaining semantically rich 3D models enables the conversion of point cloud scans into meaningful and interpretable data. The resulting HBIMs are, in any case, complementary to point clouds, 3D CAD models or non-automated high-level HBIMs, among other architectural representations, ultimately enhancing the knowledge about the building.

The challenge in automating the reconstruction of heritage building models is to deal with their geometrical complexity and irregularity. Although most of the reconstruction techniques have been applied to modern buildings, their use can be extended to heritage buildings, but they should be robust enough to deal with the complex and irregular shape. 
This book chapter is organized in five sections, starting with the current introduction. Section 2 reviews the use of laser scanning for BIM reconstruction. Section 3 describes a set of simple approaches towards the reconstruction of building façades to energy analysis purposes while Section 4 deals with the results obtained from applying the methodology to a particular case study: the Paço dos Duques in Guimarães (Portugal). Finally, Section 5 concludes this work.

\section{From Laser scanning to BIM reconstruction}

The BIM creation process differs if it is aimed towards an existing building or to a new building. Volk, Stengel and Schultmann (2014) schematize the BIM model creation process for new buildings and existing buildings depending on the availability of pre-existing BIM and according to the Life Cycle (LC) stages of a building following ISO 22263:2008. Figure 1 shows a scheme wherein BIM models for new buildings might not initially be available and they need to be created from the first step of the building LC. EBIM are typically created to support maintenance and deconstruction stages and HBIM is included in this group as a particular case of EBIM.

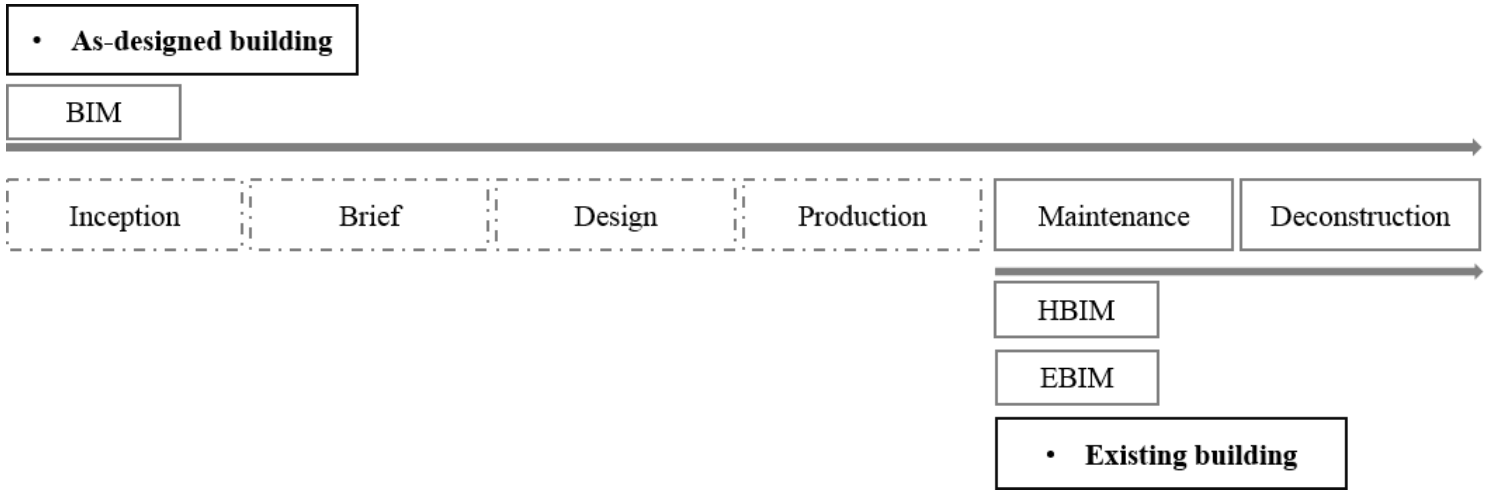

Figure 1. The creation process of BIM, EBIM and HBIM related to the Life Cycle stages following ISO 22263:2008 (Adapted from Volk, Stengel and Schultmann, 2014).

Heritage Building Information Modelling involves a reverse engineering solution, commonly named as "scan-to-BIM", whereby architectural elements are converted into parametric objects from data acquired with surveying techniques such as laser scanning and photogrammetry (Murphy, McGovern and Pavia, 2009). The development of the laser scanning technology in the recent years, together with the increased accessibility to the use of this technology, as well as its cost reduction, have made the creation of as-built BIMs tractable, assister by such technology (Tang et al., 2010). The use opportunities of laser scanners for BIM reconstruction have been evidenced by several agencies and organizations, such as the General Services Administration through its program "3D-4D Building Information Modeling" (GSA, 2009) or even Building Smart Finland through COBIM (BuildingSmartFinland, 2012). Both programs include specific recommentations about laser scanner surveying for BIM reconstruction, where different aspects of the acquisition process are discussed such as the overlapping between scans, point cloud accuracy and spatial resolution, among others. Other remote sensing techniques such as thermography can be used to extract semantic information such the thermal properties of the building materials (Lagüela et al., 2013).

Most of the processes carried out to generate HBIM consist of mapping architectural elements onto laser scan or photogrammetric survey data using a BIM software such as Autodesk Revit and Navisworks, 
Bentley Architecture, Graphisoft Archicad or Tekla (Fai et al., 2011; Oreni et al., 2014, Brumana et al., 2013). Point clouds and images are used as templates, and consequently, HBIMs are manually modelled by placing, positioning and extruding architectural elements. There is the need to streamline EBIM/HBIM reconstruction by using semi-automated and automated techniques (Tang et al., 2010). Intense efforts have been made in recent years to automate the EBIM reconstruction. Specialized software in the area of reverse engineering incorporates developed tools to allow the rapid generation of BIM components from point clouds. Nonetheless these solutions are far away from automated or semi-automated (Volk, Stengel and Schultmann, 2014).

Although literature on point cloud processing is extensive (Paatraucean et al., 2015), the issue is still an ongoing research topic because of the high variability of the building environment. In comparison with modern buildings, heritage buildings are characterized by their singularity, comprising irregular and complex components. Indeed, very little work has been done in terms of automatic HBIM reconstruction. In this context, the Duraark project aims to generate semantically rich as-built 3D models for the LongTerm Preservation of the architectural knowledge (Duraark project, 2015). They have developed several prototypes towards the automation of BIM reconstruction from point cloud scans. Similar to the approach of Adan and Huber (2011), the first prototype consisted on segmenting the point cloud into rooms and detecting doors by searching from visibility line-of-sights between rooms (Ochmann et al., 2014). A second prototype improved the method whereby walls were reconstructed including doors and windows (Ochmann et al., 2016). In all cases, point clouds were submitted to rules in order to correctly parameterize the building components, causing the loss of architectural details. In this regard, the project also proposes the joint indexing of the various types of arquitectural data ranging from point clouds to 3D CAD models and BIMs since all of them are complementary to the knowledge about the building. Other works deal with these issues by texturizing the models with real-colour images for visualization (Xiao and Furukawa, 2012) or with thermal images for energetic purposes (Lagüela et al., 2013).

\section{Methodology}

In this section, a set of simple techniques for processing point clouds towards the automation of HBIM reconstruction for energy analysis purposes is presented. Figure 2 shows the general workflow of the methodology. It starts by segmenting the point cloud into planar regions in order to identify and to model the building envelope (Section 3.1.). Then, the planar regions corresponding to walls are rasterized with high resolution in order to detect openings, such as windows and doors (Section 3.2.). Finally, the data extracted are represented according to the gbXML schema (Section 3.3.), which can be complemented with attributes obtained from other sources. 


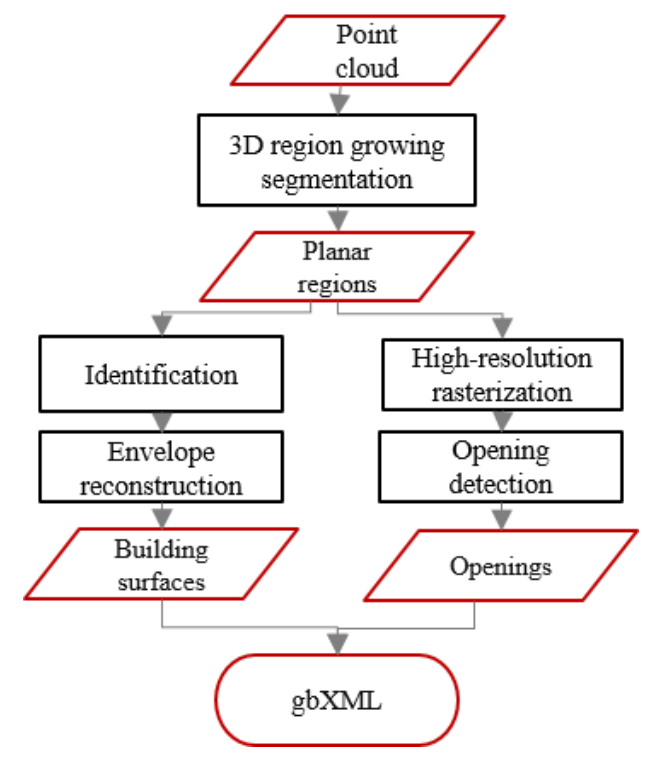

Figure 2. General workflow of the proposed methodology.

\subsection{Façade segmentation}

The proposed methodology is classified as top-down because it starts by selecting and parameterizing the general building elements such as walls. After such parameterization, a further refining is made towards the identification and modelling of more detailed elements such as, in this case, façade openings.

The complete point cloud is segmented into planar regions by implementing a seeded region-growing algorithm similar to the one used by Rabbani, Heuvel and Vosselman (2006). The point cloud is submitted to a curvature study for which the normal vector of each point is calculated by a Principal Component Analysis (PCA) using the covariance method presented by Joliffe (2002). The eigenvector associated to the smallest eigenvalue can be assumed as the normal vector of the point. The study is performed by analysing each point supported by a neighbourhood of its 50 closest points, as in Díaz-Vilariño et al. (2013). The algorithm choses the point with the lowest curvature value as a region seed candidate. In each iteration, the algorithm analyises the point cloud and includes all points satisfying two geometric conditions: planar fitting referring to the distance $\left(d_{p}^{r}\right)$ between the point $(\mathrm{p})$ and the planar region (r); and surface smoothness related to the angular difference $(\alpha)$ between the direction of its normal vector $\left(\mathrm{n}_{\mathrm{p}}\right)$ and the region normal $\operatorname{vector}\left(\mathrm{n}_{\mathrm{r}}\right)$ (Figure 3).



Figure 3: The geometric conditions to consider a point $(\mathrm{p})$ included in a planar region. 
Given the irregular and complex elements of heritage buildings, segmentation thresholds $\left(d_{p}^{r}\right.$ and $\left.\alpha\right)$ should be broad enough to ensure that façade points are classified as inliers in the segmentation process. For example, if the historic wall is built using non-plastered masonry, the mortar joints have different depths, being an intrusion from the wall plane. Moreover, historic walls are usually out-of-plumb and exhibit important curvature. While it is interesting to keep small intrusions conforming the wall region, this planar fitting parameter can be useful for not including large protrusions such as those corresponding to openings.

Once the point cloud is segmented into regions that correspond to façade surfaces, they should be identified and intersected with roofs and floor in order to obtain the boundary points that enclose the points of façade planes. Figure 4 shows an example of this process by which segmented points describing a façade surface are enclosed by the boundary points obtained from the plane-to-plane intersection.

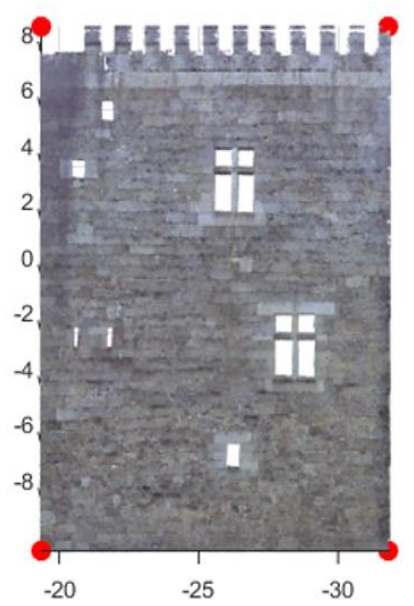

Figure 4. Image of a segmented façade region visualized in real colour and the extracted boundary points enclosing them, visualized in red colour.

\subsection{Reconstruction of openings.}

One of the most relevant challenges of modelling heritage elements is to deal with irregularity. Openings are commonly placed in façades following an irregular distribution as a result of the changes and renovations of the heritage building over time. Nonetheless, openings are generally characterized by being placed at a certain depth from the wall plane (intrusions) and, for this reason, they can be considered as holes in the wall, which in turn means areas without points after a planar segmentation process.

In a similar fashion to the work reported by Díaz-Vilariño et al. (2015b), the method to reconstruct windows and doors is based on the Generalized Hough Transform (GHT) and it consists on finding parametric shapes in edge images. Although a large variety of shapes can be parameterized (Khoshelham, 2007), rectangles are the most common shape for doors and windows.

The crucial step in this process is the correct generation of the edge images used as input for the GHT. Better edge images result in faster and more accurate results. In the approach of Díaz-Vilariño et al. (2015b), edge images were obtained from the reconstruction of real-colour wall images, since closed doors might be coplanar with the walls to which they belonged. In the work reported in this Chapter, openings such as doors and windows are considered as holes in the point cloud, so no colour information is taken into account, and the edge images are obtained from raster wall images. 
Each façade region is converted into a raster image by projecting the points on the wall plane defined by the façade boundary points. With this purpose, a rectangular matrix is created and each pixel is assigned with value 'one' if the projected point cloud fills that specific pixel, or value 'zero' otherwise. In this way, pixels corresponding to the wall are represented by 'one' (in white) and points correspondent to opening are represented by 'zero' (in black) as shown in Figure 5, a. If the raster image is created by assigning the value of point depth with regard to a reference point, the singularities of the façade can be represented; according to the image in Figure 5, b. This second approach that represents singularities is outside the scope of this chapter. An alternative way to create a raster would consist in considering the intensity value of each point of the cloud returned by the laser scanner. In this regard, Riveiro et al. (2015) used the reflective intensity of laser light to isolate masonry blocks in the Guimarães Castle (Portugal).

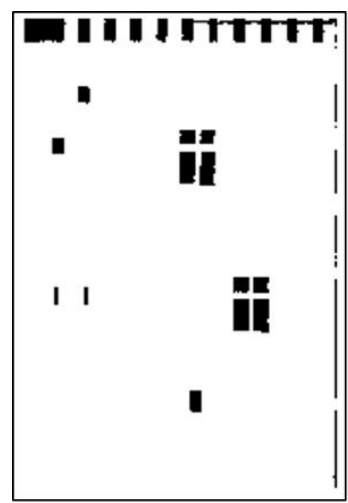

(a)

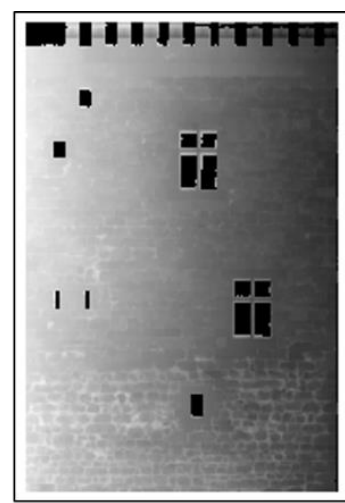

(b)

Figure 5: Image a represents rasterization considering the absence or presence of points Image b shows rasterization taking into account the depth of the points with regard to the most external region point (according to the normal). In this case, mortar areas are distinguished from masonry units especially in the lower part.

For a successful rasterization, wall regions are previously rotated around Z-axis in a way that they are parallel to $\mathrm{XZ}$ or $\mathrm{YZ}$ axis of the point cloud coordinate system, as appropriate (Díaz-Vilariño et al, 2015a). Finally, the binarized image is submitted to edge detection using the Canny method (Canny, 1986), based on finding image gradients.

The parameters involved in the detection process are represented in Figure 6. For each image, the origin of the xy coordinate system is established in the lower left pixel. The detection of rectangular candidates is carried out in two steps. Firstly, an R-table is created where the shape of a rectangle is stored and represented by its edge orientation $(\theta \mathrm{i})$ and a vector defined $(\mathrm{r}, \beta)$ to an arbitrary centre point. Next, the orientation of each edge pixel $(\theta \mathrm{i})$ is calculated and used to find the corresponding vector in the R-table. Using the vector and pixel coordinates, a center point is reconstructed and used to cast a vote in a 4D Accumulator Array ( $a$, $b, S x, S y$ ), where $a$ and $b$ are the coordinates of the centre point, and $S x$ and $S y$ are the scale parameters of the shape, corresponding to width and length of the rectangle.

Rectangles are selected by searching for maximum peaks (votes) in the accumulator array. Furthermore, the detection is enforced to a maximum and minimum opening width and height and overlapping rectangles are not allowed. 


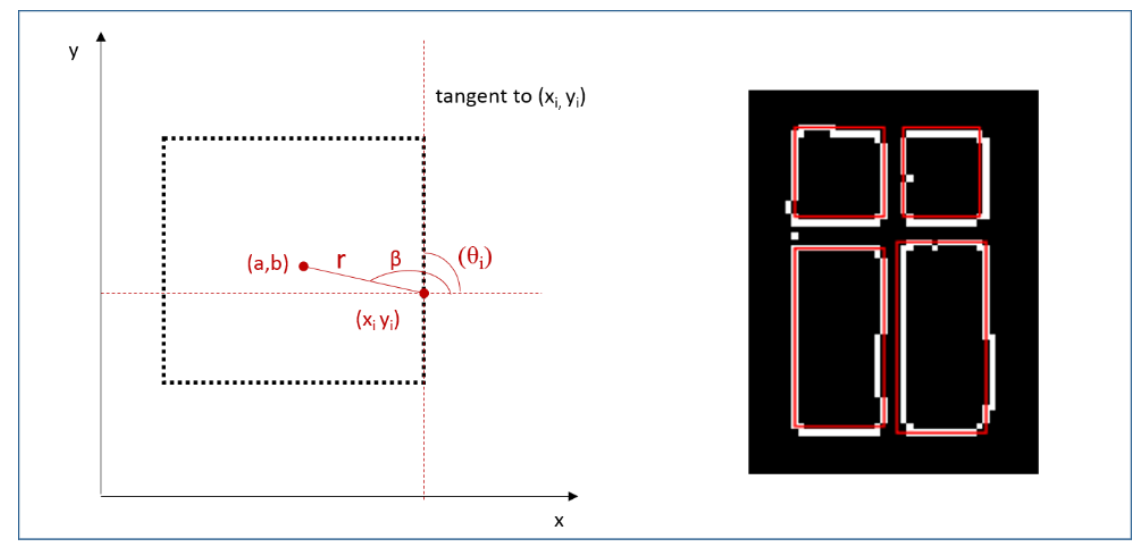

Figure 6: Parameters involved in the GHT (left); result of the application of GHT to an opening modelling from the façade represented in Figure 4 (right).

\section{3. gbXML generation}

The final step of the proposed methodology is the conversion of the extracted geometric data into a BIMbased schema for energy analysis: the Green Building XML (gbXML).

gbXML represents buildings with their geometry, semantics, topology and appearance. Because gbXML is based on the XML language (Xtensible Markup Language), it is structured as a tree of objects/elements with attributes. In Figure 7, the parent/child relationships among elements are represented for illustrative purposes. Geometry is contained in the "Campus" element and "Opening" elements is a child of "Surface" element. As shown in Figure 7, semantic contents such as construction, layer or materials are related to the corresponding geometry by the element attributes (e.g. "id", etc.) (gbXML schema).

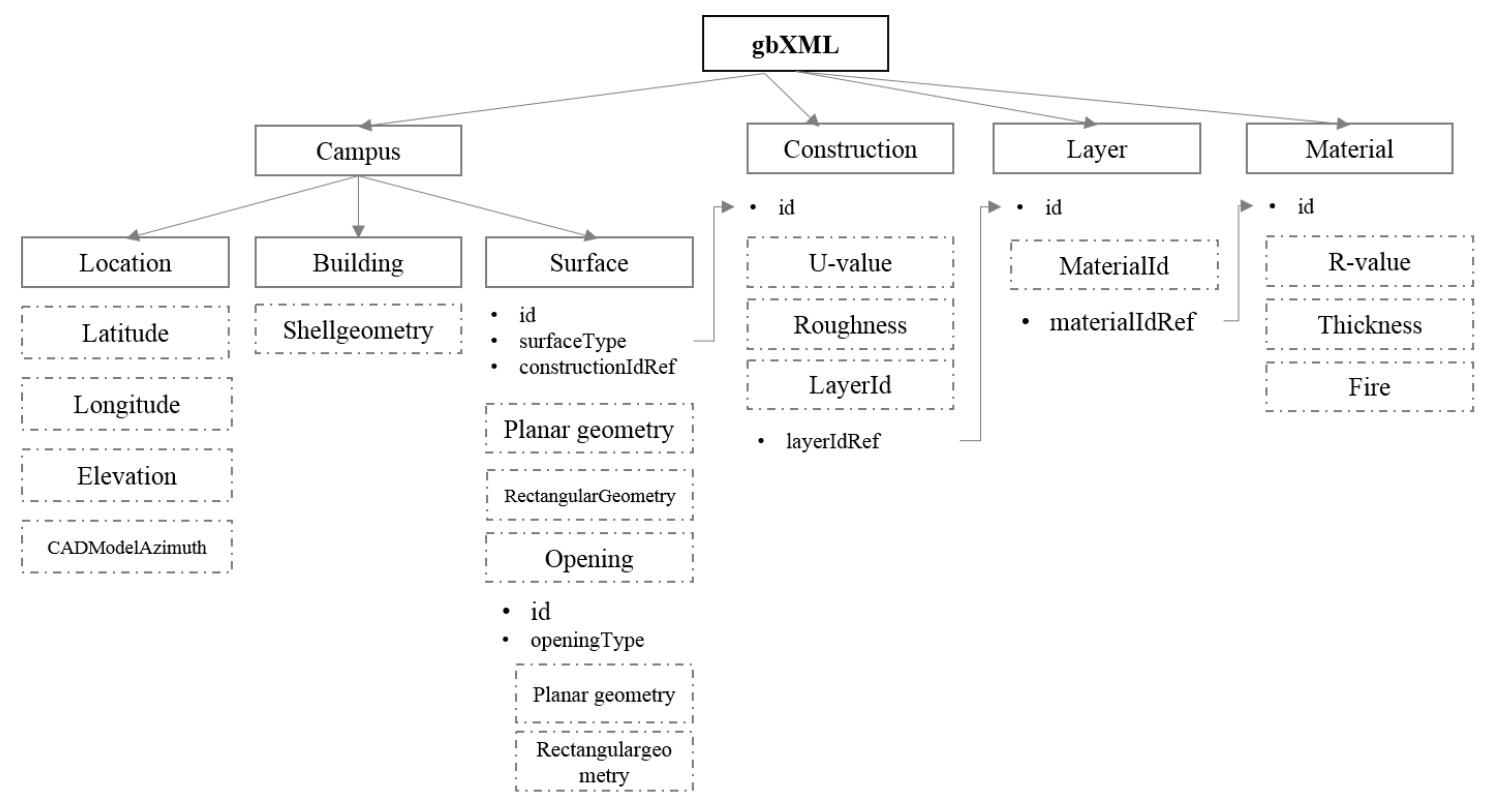

Figure 7. gbXML structure showing the relationship between the elements represented with contours and attributes represented with black dots.

The schema was developed to support energy analyses, whereby surfaces are represented as simple geometries. Walls, ceilings and floors are normally modelled as planar surfaces, in most cases represented by four boundary points in their three-dimensional representation, given by its three Cartesian coordinates 
( $\mathrm{x}, \mathrm{y}$ and $\mathrm{z}$ ). A curved wall should be modelled as a set of continuous planar polygons. Openings need to be represented as rectangles coplanar with the wall to which they belong. These are just some of the requirements that have to be taken into account for modelling an existing building, whether being heritage or not, if the purpose is to adopt it for energy analysis, wherein parameterization provides a simplification of the building geometry. The model can be complemented with semantic attributes, such as those corresponding with materials of which they are made and their thermal properties such as R-value, thickness, conductivity, etc. As in Lagüela et al. (2014), other remote measurement instruments such as thermographic cameras can be used to assist estimations of the thermal properties of building element in a non-destructive way. Thickness could be obtained from the analysis of the point cloud if indoor rooms are acquired and registered in the same coordinate system as the façade.

Because BIM standards are mostly conceived for as-designed rather than as-built buildings, important characteristics such as thermal bridges cannot be represented in gbXML, for example. Even more, the attributes of a component are common for the whole component: the thickness value included in gbXML is unique for the entire wall and variations of a wall thickness cannot be addressed. However, the schema could be easily extended. In this context, the critical issue would be the interoperability with applications for which the models are generated.

\section{Case study}

\subsection{General overview}

The Bragança Ducal Palace (Figure 8) is located in Guimarães, in northern Portugal. Its construction began during the $15^{\text {th }}$ century, under order of the first Duque of Bragança, D. Afonso.

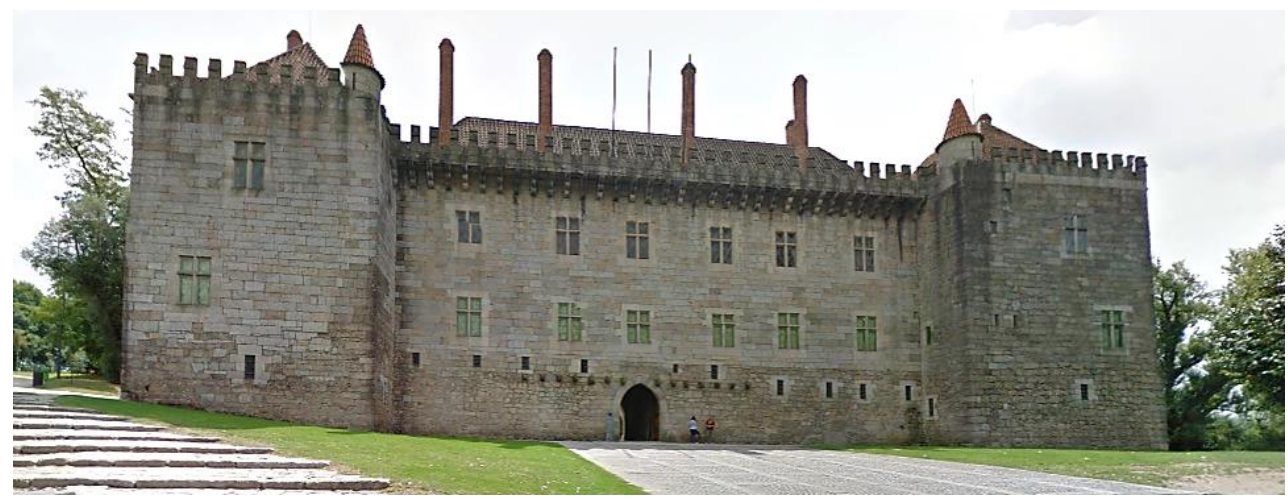

Figure 8: Image of the Palace of the Dukes of Bragança (from Google Maps).

The Palace was only permanently inhabited during the $15^{\text {th }}$ Century, and was then progressively abandoned until it became a ruin. Afterwards, in the $19^{\text {th }}$ century, the Palace was used for military purposes. The renovation of the Palace was carried out between 1937 and 1959 based on a project by the architect Rogério de Azevedo. Currently, the Ducal Palace is classified as a National Monument, and it involves a museum at the $1^{\text {st }}$ floor, as well as a specific quarters in the $2^{\text {nd }}$ floor assigned to the President of the Republic.

Besides its historical importance, the Palace is one of the most visited Museums in Portugal. Due to its characteristics and geometric complexity, it was selected as a case study to assess the feasibility of the proposed methodology. 


\subsection{Instruments and data}

The survey of the Palace was carried out with a Terrestrial Laser Scanner FARO Focus3D X 330 with the technical characteristics summarized in Table 1.

Table 1. Technical characteristics of the FARO Focus3D X 330 laser scanning device according to the manufacturer datasheet (Faro Focus 3D X 330 Tech Sheet, 2013).

\begin{tabular}{ll}
\hline Technical Characteristics & \\
\hline Measurement range & From $0.6 \mathrm{~m}$ to $330 \mathrm{~m}$ \\
Ranging error (25m, one sigma) & $\pm 2 \mathrm{~mm}$ \\
Step size (Vertical/Horizontal) & $0.009^{\circ} / 0.009^{\circ}$ \\
Field of view (Vertical/Horizontal) & $300^{\circ} / 360^{\circ}$ \\
Beam divergence & $0.011^{\circ}$ \\
Measurement rate (points per second) & $122.000-976.000$ \\
Laser wavelength & $1550 \mathrm{~nm}$ \\
\hline
\end{tabular}

Data was acquired from different scan positions in order to cover the entire area of interest. Ten scans were carried out in order to complete the interior and exterior façade survey and they were placed to ensure an overlapping between consecutive stations, enabling the registration into the same coordinate system by manually selecting common reference points. Although colour is not neccessary for implementing the methodology, it was acquired to ease the recognition of singularities in the point cloud, such as corners or windows, which are used as reference points between adjacent scans. Figure 9 shows the surveying strategy carried out for acquiring the complete façade of the Palace taking into account its dimensions and its surroundings. Roofs and buried parts of the Palace are not acquired.

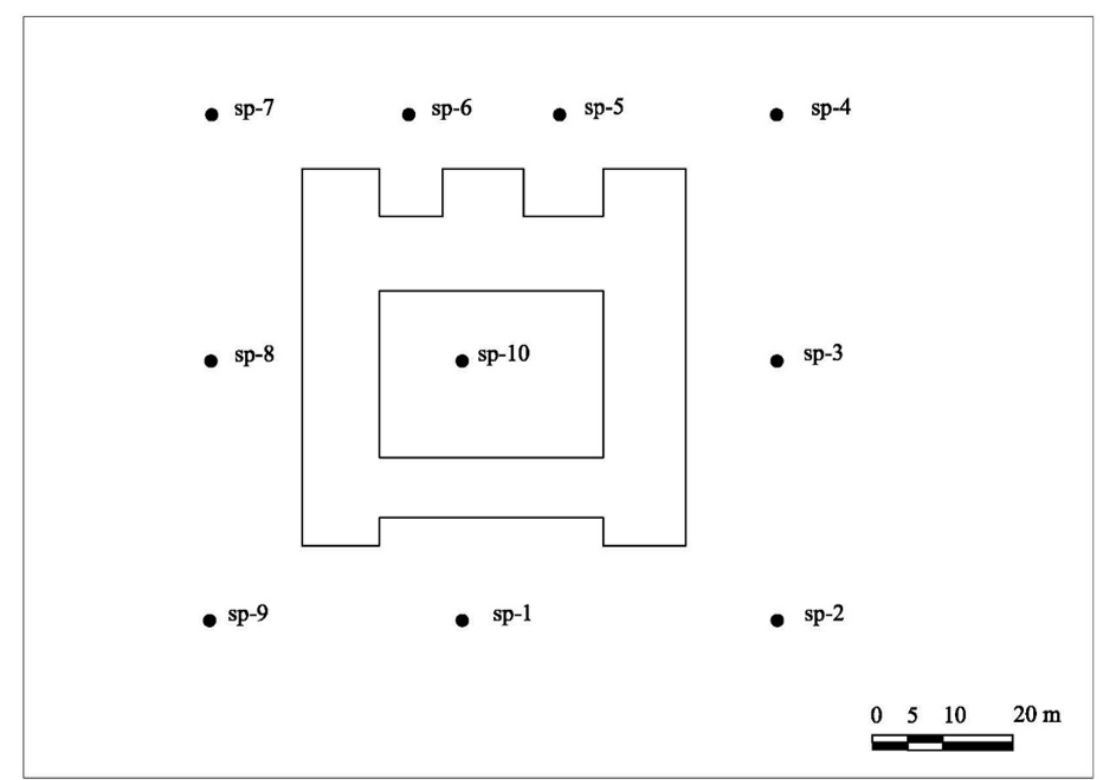

Figure 9: Schema of the surveying strategy for this case study.

Points corresponding to exterior elements that do not belong to the Palace itself, such as trees, grass, outer walls, etc. were deleted from the point cloud in order not to consider them for subsequent steps. Furthermore, the point cloud is submitted to an octree filter (Meagher, 1982) to ensure uniform density. 
Octree parameters are $0.15 \mathrm{~m}$ for $\mathrm{X}, \mathrm{Y}$, and $\mathrm{Z}$ directions and resulting point cloud contains 441.075 points. This process was carried out in Riscan Pro software. Figure 10 shows the point cloud obtained from registering all scans and cleaning exterior elements not belonging to the building. The origin of the coordinate system is established in the origin of scan position 1 (sp-1 in Figure 10).

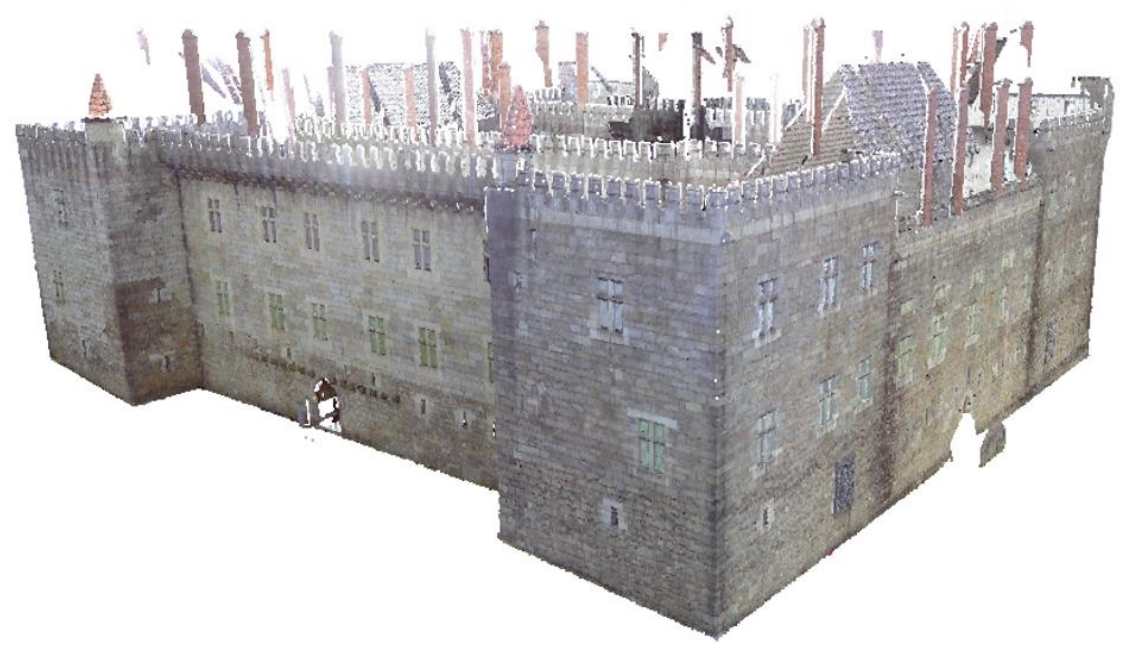

Figure 10: Point cloud of the complete façade of the Palace, displayed in true-colour.

\subsection{Processing results}

As explained in Section 3.1., segmenting the point cloud is the first step of the methodology. The segmented regions are the basis for the rasterization, so this step should be carefully addressed. Due to the large size of the point cloud, a two-step segmentation was implemented in this case study. Firstly, a rough segmentation was carried out to create general regions and to obtain the limits of the façades. In this case, angular and distance point-to-plane thresholds were $45^{\circ}$ and $0.5 \mathrm{~m}$ respectively resulting into 553 regions. Regions were selected according to a minimum region size (5000 points) resulting into 21 regions, which were classified in façades and floor according to their normal (Figure 11).

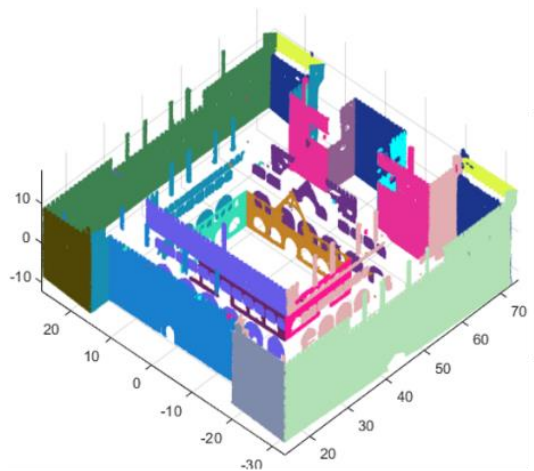

(a)

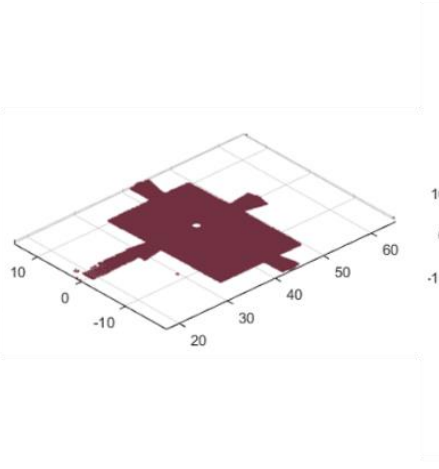

(b)



(c)

Figure 11: Planar regions are classified as walls (image a), floor (image b) and remaining regions (image c) [Units: $\mathrm{m}$ ].

Secondly, a fine segmentation was implemented to refine wall regions. The objective was to deselect all the points that actually do not belong to the wall surface, such as window glasses, reflections or window fences. For the purpose of obtaining a higher point density for creating the raster images, the information 
of the previous segmentation (i.e. definition of the location of each wall) was used for selecting the new input data from sub-sets of original point cloud with density of 1 point/0.01m for X, Y, and Z directions of the point cloud coordinate system. The angular threshold and distance point-to-plane were $45^{\circ}$ and $0.10 \mathrm{~m}$, respectively, in this case. With these conditions, if walls are slightly curved due to their large size, construction sequence or historic alterations, points far away from the centroid might fail in the point-toplane condition. To avoid this, the fine segmentation was processed by using a mask of $5 \mathrm{~m}$ length along an horizontal plane, and covering the full height of the wall. An overlapping of $0.5 \mathrm{~m}$ was considered between adjacent masks.

From the refined wall regions, raster images were created with $0.06 \mathrm{~m}$ resolution. In the rasterization process, a pixel is considered as occupied if at least four points from the cloud point are inside the pixel (being density of the point cloud 1 point $/ 0.01 \mathrm{~m}$ ). This condition was established for avoiding falsely occupied pixels caused by the existence of one isolated point. Afterwards, the binarized raster is processed by the Canny edge detector. Figure $12 \mathrm{~b}$ shows the rasterization result of the point cloud corresponding to the main façade (Figure 12 a) and Figure 12 c corresponds to the edge image of Figure $12 \mathrm{~b}$.

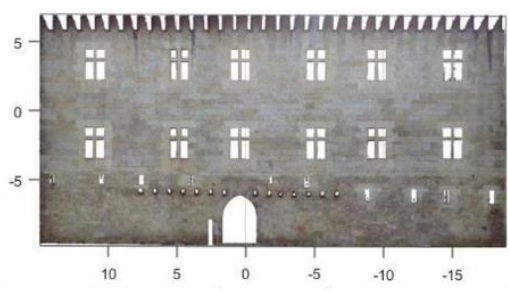

(a)

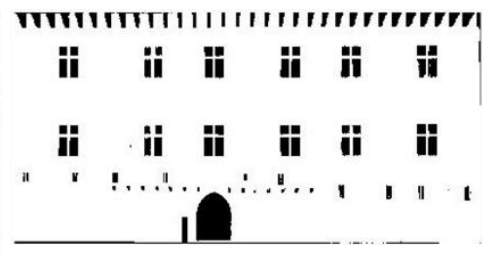

(b)

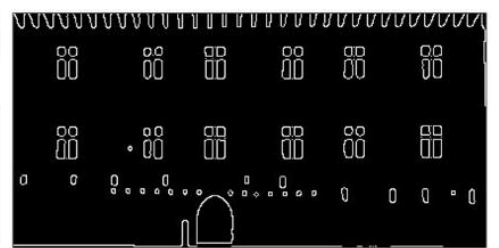

(c)

Figure 12. Main façade of the Ducal Palace: a) point cloud; b) raster image; c) edge image [Units: m].

In this case, no morphological operations were implemented to improve the quality of the raster since point cloud density was sufficient to adequately fill all wall pixels at the resolution of $0.06 \mathrm{~m}$. Resolution should limited while ensuring that enough points are available to obtain a good edge image quality (Figure 12 c). This strategy reduces the computational time and improves the quality of the openings detected, as openings with noisy edges are more difficult to detect by the GHT.

In the final step, the edge image is used in a rectangle search based on the GHT. As the number of rectangles is not known, a large number of peaks are searched and opening candidates are selected by their vote ratio. Furthermore, the analysis is enforced to a minimum and maximum opening width and height. Figure 13 represents the results of an opening detection enforced to a minimum/maximum width and height of $130 \mathrm{~cm} / 200 \mathrm{~cm}$ and $160 \mathrm{~cm} / 250 \mathrm{~cm}$, respectively. As the number of openings is assumed as unknown, a high number of peaks is searched (50 peaks), and then, opening candidates are selected if their voting percentage is above the 75 percentile. 


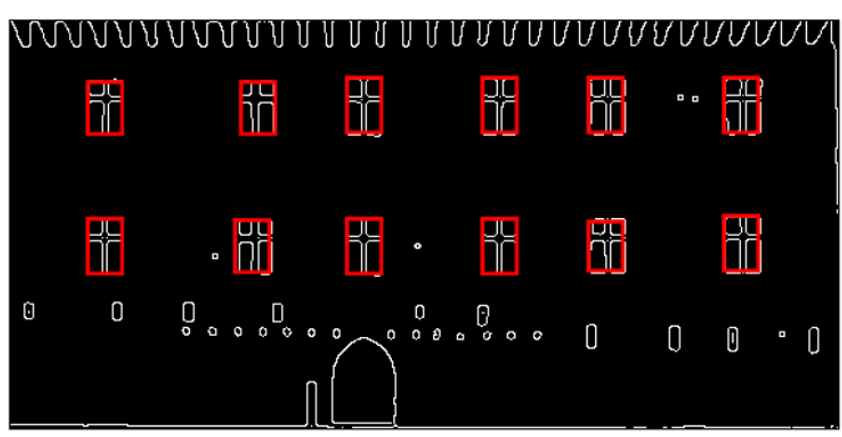

Figure 13. Results of applying the GHT to the main wall.

In this wall no false positives were detected but they may be obtained in the presence of similar elements in size and shape to the objects that are being searched (Díaz-Vilariño et al., $2015 \mathrm{~b}$ ). With regard to false negatives, they can be obtained if previous steps produce a bad-quality edge image. For instance, a noisy edge can be caused by a bad segmentation due to the co-planarity of a window with the wall where it is contained, or due to the presence of additional structures such as window fences. The search can be extended to other parametric shapes and enforce the detection with different rules. For instance, if one wants to detect a door, the y coordinate of the centroid should be positioned to a certain distance from the floor. Finally, image coordinates of the openings are reprojected to obtain the $3 \mathrm{D}$ coordinates necessary to reconstruct the gbXML (Díaz-Vilariño et al., 2015 b).

\section{4. $g b X M L$}

Once the geometry of the parameterized components has been extracted, it is represented according to the gbXML schema (Figure 6). The global position of the building is defined in the element "Location", which is completed with "Latitude", "Longitude" (geographic coordinates in decimal degrees), "Elevation" (in meters) and "CADModelAzimuth" (the orientation from North). The "Building" element defines volumes enclosed by surfaces, typically indoor spaces. Each surface is represented by the "Surface" element which is defined by several attributes that provide semantic information to the object, e.g.: attributes "id" or "surfaceType" attached to "ExteriorWall", "Roof", "Shade", "RaisedFloor", etc. Figure 14 (right) represents a fragment of the gbXML schema, wherein some elements such as "Building", "Surface" or "Openings" are shown.

The semantically rich 3D model can be used for energy analysis. The image a from Figure 14 shows a capture of the model directly imported into Sketchup by using the plugin Openstudio from Energyplus, wherein the recognition of semantic attributes puts in value the resulting model with regard to other nonsemantic models. On the other hand, image b shows the model texturized with images from the point cloud, representing different complementary levels of knowledge about the building. 


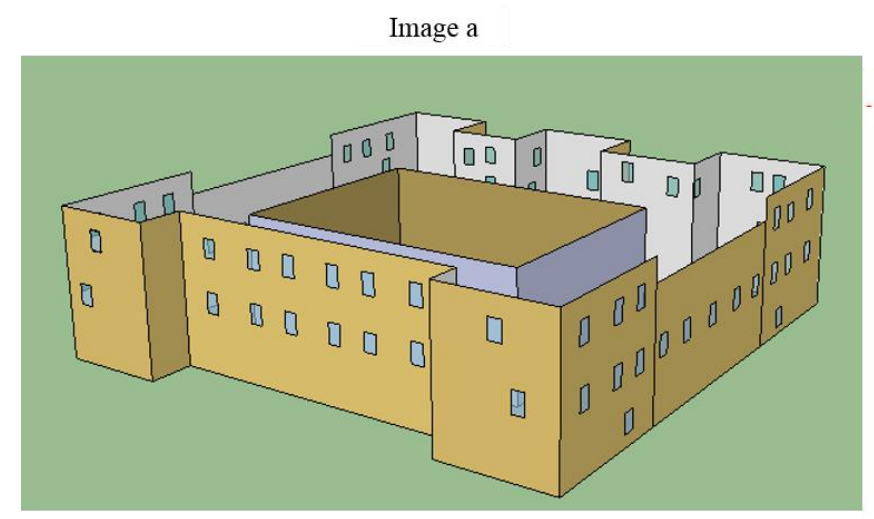

$<? \times$ xml version=" 1.0 " encoding="UTF-8"?

gbXML volumeUnit="CubicMeters" version="0.37"

useSIUnitsForResults="true" temperatureUnit="C" lengthUnit="Meters"

areaUnit="SquareMeters"

xmlns="http:// www.gbxml.org/schema" >

<Campus id="cmps-1">

<Location>
$<$ Building id="bldg-1" buildingType="Unknown">

<BuildingStorey id="bldg-stry-1">

$<$ Name $>$ Nivel1 $</$ Name $>$

$+<$ Space id $="$ "sp-1"

spaceType $=$ "UnknoneIdRef =" $\mathbf{z n - 1}$

$</$ Building >

$<$ Surfacid

$<$ Adjad

$+<$ RectangularGeometry $>$

+ <Opening id $=$ "op-1" openingType="NonslidingWindow"

$+<$ Opening id $="$ op-3" openingType $=$ "NonSlidingWindow"

$+<$ opening id $=$ "op-4" openingType="NonslidingWindow"

+ $\langle$ Opening id="op-5" openingType="NonSlidingWindow"

+ <opening id $="$ "op-6" openingType="NonSlidingWindow"

+ <Opening id="op-7" openingType="NonslidingWindow"

Image b

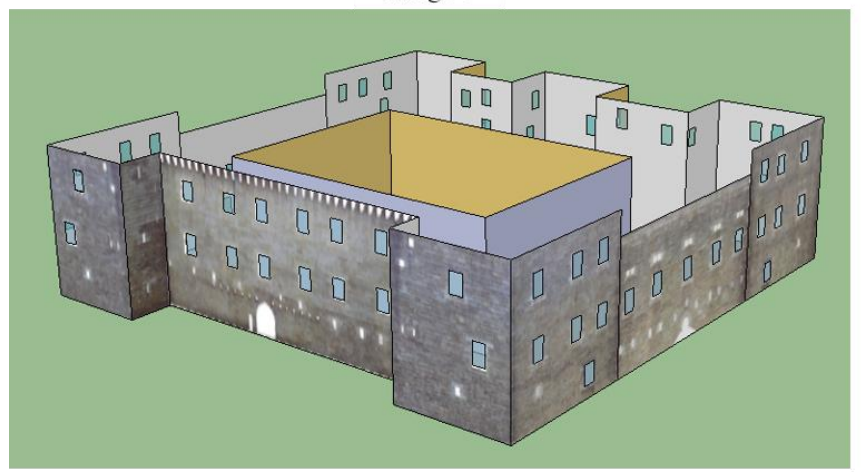

$+<$ Opening id $="$ "op-8" opening Type="NonSlidingWindow"

$+<$ opening id $="$ op-9"

openingType $=$ "NonSliding Window"

$+<$ Opening id =" op-11"

openingType $=$ "Nonsliding Window"

$+<$ Opening id $="$ op -12

openingType $=$ "NonSlidingWindow"

$</$ Surface $>$

- < Surface id="su-2" surfaceType="ExteriorWall" >

+ < Surface id="su-3" surfaceType="ExteriorWall" $>$

<Surface id ="su-5" surfaceType= "ExteriorWall">

<Surface id="su-6" surfaceType="ExteriorWall">

- < Surface id="su-7" surfaceType="ExteriorWall">

$+<$ Surface id="su-8" surfaceType="ExteriorWall">

$<$ Surface id="su-9" surfaceType="ExteriorWall">

$+<$ Surface id $=$ "su-10" surfaceType $=$ "ExteriorWall"

+ <Surface id="su-11" surfaceType="ExteriorWall"

- <Surface id= su-12 suffaceType= ExteriorWall"

Csurace id="su-14" surfaceType="ExteriorWall">

Figure 14: The gbXML schema is directly imported into Sketchup using the Openstudio plugin. Image a shows the model without texture and image $\mathrm{b}$ shows the model texturized with images from the point cloud (the roof has been deleted for visualization) (left). Fragment of the gbXML where windows from the main façade (Figure 12) are represented (right).

\section{Conclusion}

This chapter deals with the use of laser scanning for documenting the real geometry of a heritage building and the use of automated techniques to parameterize the point cloud in order to define building façade elements according to energy analysis specifications.

Although laser scanners allow the accurate and efficient 3D digitalization of heritage, point clouds should be parameterized to create sets of components. The parameterization required for energy analysis software is highly specific since building elements should be represented as rectangular polygons. This form of representation is usually far from the reality, especially in case of heritage buildings, which are often characterized by singularities associated to the architectonic style, the traditional way of construction and renovations over time. Methodologies and results presented in this chapter are not suitable for visualizing and documenting the heritage and cultural value of building elements. However, despite the fact that they do not represent a BIM in the broader sense, they represent a BIM (and particularly HBIM) in the narrow sense since the results fit with the purpose of modelling the heritage building for a specific application.

From the processing methodology, the following specific conclusions can be drawn:

- Segmentation parameters should take into account the irregularity of heritage building components for ensure a complete segmentation of wall façades. 
- Rasterization resolution depends on the point cloud density for obtaining good quality edge images.

- Higher resolution implies higher computational time and poses difficulties to the algorithms for detection of openings.

- After the first coarse segmentation, a second finer segmentation is necessary for deselecting windows and other openings. Noisy window edges make their detection more difficult, causing false negatives.

- Several parametric patterns can be used for detecting objects with similar shape in edge images. False positives can be detected in case of the presence of other objects with similar size and shape.

The overall conclusion of this chapter is that simple automatic techniques for processing point clouds can be used in heritage buildings for creating a BIM model if the purpose is to parameterize and simplify the real geometry, according to the requirements of the application it is going to be submitted. The resulting 3D semantically rich model enhances the knowledge of the heritage building, complementing other representations of the facility such as point clouds or hand-made HBIM.

\section{Acknowledgements}

Authors would like to thank to the Ministerio de Economía y Competitividad (Gobierno de España), for the financial support given through human resources grant (FPU AP2010-2969, ENE2013-48015-C3-1-R). This work is also partly supported by FEDER funds through the IACOBUS program and by FCT (Portuguese Foundation for Science and Technology), within ISISE, project UID/ECI/04029/2013. The authors also like to thank all the institutions and companies that have been involved supporting and contributing for the development of this study, in particular, Isabel Fernandes and Flávio Vieira, Director and staff member of Bragança Ducal Palace. Research of the second and fourth author is supported by a National Priority Research Program NPRP award (NPRP-06-1208-2-492) from the Qatar National Research Fund (a member of The Qatar Foundation). The statements made herein are solely the responsibility of the authors.

\section{References}

Adan, A., Huber, D 2011, 3D reconstruction of interior wall surfaces under occlusion and clutter. 3D Imaging, Modeling, Processing, Visualization and Transmission (3DIMPVT), 16-19 May 2005, 275-281.

Autodesk. 2003. Autodesk BIM white paper [online] Available at: $<$ http//www.laiserin.com/features/bim/autodesk_bim.pdf> [Accessed 14 June 2015]

Bosché, F., Ahmed, M., Turkan, Y., Haas, C.T., Haas, R. 2015. The value of integrating scan-to-bim and scan-vs-bim techniques for construction monitoring using laser scanning and bim: the case of cylin-drical MEP components. Automation in Construction, 49, pp.201-213.

Brumana, R., Oreni, D., Raimondi, A., Georgopoulos, A., \& Bregianni, A. 2013. From Survey to HBIM for Documentation, Dissemination and Management of Built Heritage: The Case Study of St. Maria in 
Scaria d'Intelvi. Proceedings of the $1^{\text {st }}$ International Congress of Digital Heritage. Marseille, France, 28 October -1 November 2013. pp. 497-504.

Canny, J., 1986. A Computational Approach to Edge Detection. IEEE Transactions in Pattern Analysis and Machine Intelligence, 8, pp. 679-698.

Díaz-Vilariño, L., Conde, B., Lagüela, S., Lorenzo, H., 2015. Automatic Detection and Segmentation of Columns in As-Built Buildings from Point Clouds. Remote Sensing, 7, pp.15651-15667 (a)

Díaz-Vilariño, L., Khoshelham, K., Martínez-Sánchez, J., Arias, P. 2015. 3D modelling of building indoor spaces and closes doors from imagery and point clouds. Sensors, 15, pp.3491-3512. (b)

Díaz-Vilariño, L., Lagüela, S., Armesto, J., Arias, P., 2013. Semantic as-built 3D models including shades for the evaluation of solar influence on buildings. Solar Energy, 92, pp. 269-279.

Eastman, C., Teicholz, P., Sacks, R., Liston, K. 2008. BIM Handbook, A Guide to Building Information Modeling for Owners, Managers, Designers, Engineers, and Contractors, Hoboken, N.J.: John Wiley \& Sons

Fai, S., Graham, K., Duckworth, T., Wood, N., Attar, R., 2011. Building Information Modeling and Heritage Documentation. Proceedings of the XXIIIrd International CIPA Symposium. Prague, Czech Republic, 12-16 September 2011.

FARO Laser Scanner Focus 3D X 330 Tech Sheet, 2013. Available at: <http:// http://www.faro.com/> [Accessed 20 December 2015].

GbXML schema, 2015. Available at: < http://www.gbxml.org/ > [Accessed 20 December 2015].

Isikdag, U., Zlatanova, S., Underwood, J., 2013. A BIM-Oriented Model for supporting indoor navigation requirements. Computers, Environment and Urban Systems, 41, pp.112-123

Joliffe, I.T., 2002. Principal component analysis. Series in Statistics, 28, p. 487. Springer

Khoshelham, K., 2007. Extending generalized Hough transform to detect 3D objects in laser range data. Proceedings of the ISPRS Workshop on Laser Scanning and SilviLaser. Espoo, Finland, 12-14 September 2007, 36, pp. 206-210

Lagüela, S., Díaz-Vilariño, L., Martínez-Sánchez, J., Armesto, J., 2013. Automatic thermographic and RGB texture of as-built BIM for energy. Automation in Construction, 31, pp.230-240

Lagüela, S., Díaz-Vilariño, L., Armesto, J., Arias, P. 2014. Non-destructive approach for the generation and thermal characterization of an as-built BIM. Construction and Building Materials, 51, pp.55-61

Meagher, D. 1982. Geometric modeling using octree encoding. Computer Vision Graphics and Image Processing, 19, pp. 129-147.

Murphy, M., McGovern, E., Pavia, S., 2009. Historic Building Information Modelling (HBIM). Structural Survey. 27 , pp. $311-27$. 
Ochmann, S., Vock, R., Wessel, R., Tamke, M., Klein, R. 2014. Automatic generation of structural building descriptions from 3d point cloud scans. Procedings of the International Conference on Computer Graphics Theory and Applications. Lisbon, Portugal, 5-8 January 2014.

Ochmann, S., Vock, R., Wessel, R., Klein, R., 2016. Automatic Reconstruction of Parametric Building Models from Indoor Point Clouds. Computers \& Graphics, 54, pp. 94-103.

Oreni, D., Brumana, R., Georgopoulos, A., Cuca, B. 2014. HBIM Library Objects for Conservation and Management of Built Heritage. International Journal of Heritage in the Digital Era, 3, pp.321-334.

Patraucean, V., Armeni, I., Nahangi, M., Yeung, J., Brilakis, I., Haas, C., 2015. State of research in automatic as-built modelling. Advanced Engineering Informatics, 29, pp.162-171.

Rabbani, T., Heuvel, FA, Vosselman, G., 2006. Segmentation of point clouds using smoothness constraint. International Archives of Photogrammetry, Remote Sensing and Spatial Information Sciences, 36, pp.24853

Riveiro, B., Lourenço, P.B., Oliveira, D.V., González-Jorge, H., Arias, P., 2015. Document Automatic Morphologic Analysis of Quasi-Periodic Masonry Walls from LiDAR. Computer-Aided Civil and Infrastructure Engineering, DOI:10.1111/mice.12145

Simeone, D., Cursi, S., Toldo, I., Carrara, G., 2014. BIM and knowledge management for Building Heritage. 681-690, Available at: <http://www.academia.edu/> [Accessed 20 December 2015]

Tang, P., Huber, D., Akinci, B., Lipman R., Lytle, A., 2010. Automatic reconstruction of as-built building information models from laser-scanned point clouds: a review of related techniques. Automation in Construction, 19, pp.829-843

Volk, R., Stengel, J., Schultmann, F., 2014. Building Information Modeling (BIM) for existing buildings literature review and future needs. Automation in Construction, 38, 99. 84-91

Xiao J., Furukawa, Y., 2012. Reconstructing the world's museums. Proceedings of the 12th European Conference on Computer Vision (ECCV2012), 7-13 October 2012, pp. 668-681. 